\title{
CRITICAL REVIEW OF PHARMACOGENOMICS IN ANTIPARKINSONIAN DRUG THERAPY: IMPACT ON CLINICAL MANAGEMENT OF PARKINSON'S DISEASE
}

\author{
POORNIMA R*, SABREEN BASHIR \\ ${ }^{1}$ Department of Pharmacology, JSS Medical College, Mysuru, Karnataka, India. Email: poornimarprasad@gmail.com
}

Received: 09 June 2016, Revised and Accepted: 26 July 2016

\section{ABSTRACT}

Objective: Parkinson's disease (PD) is a chronic progressive neurodegenerative disorder characterized by rest tremors, bradykinesia, rigidity, postural instability, gait dysfunction, and several non-motor symptoms. The marked difference in drug response and adverse effect profile among patients led to search of genetic markers and polymorphism associated with response to antiparkinsonian drugs which will enable us to predict an individual's response to drugs in terms of both efficacy and toxicity. Hence, efforts to define the role of genetic polymorphism in optimizing pharmacotherapy of PD have been undertaken and some promising genetic loci for the treatment have been determined. Therefore, we aim to present a critical review of pharmacogenetic aspects of levodopa, dopamine agonists (DAs), and catecol-O-methyltransferase (COMT) inhibitors and describe gene polymorphism of interest for future research.

Methods: The PubMed database was searched using the keywords "parkinsonism," “antiparkinsonian drugs," "levodopa," "DAs," “COMT inhibitors," "pharmacogenomics," and "polymorphism." Abstracts and review articles were included for the study.

Results and Conclusion: Studies conducted in different settings suggest that pharmacogenomics plays a significant role in Parkinsonism drug therapy. The gene/drug pairings with the strongest potential for pharmacogenetic recommendations include COMT allele/levodopa and entacapone, dopamine D2 receptor (DRD2)/ropinirole, pramipexole, and DRD3/ropinirole and pramipexole. It can be concluded that with the rapid development of genotyping platforms, genome-wide association study can be performed to analyze polymorphism associated with inefficient treatment or adverse effects. Hence, individualized therapy of PD for better patient care can be achieved by targeted genetic testing.

Keywords: Parkinson's disease, Catecol-O-methyltransferase inhibitors, Levodopa, Pharmacogenomics.

(C) 2016 The Authors. Published by Innovare Academic Sciences Pvt Ltd. This is an open access article under the CC BY license (http://creativecommons. org/licenses/by/4. 0/) DOI: http://dx.doi.org/10.22159/ajpcr.2016.v9i6.13350

\section{INTRODUCTION}

Parkinson's disease (PD) is the second most common neurodegenerative disorder with increasing evidence of genetic factor contributing to its etiology. Availability of effective pharmacological therapy distinguishes it from other neurodegenerative disorders. The nigrostriatal dopaminergic pathway is the target for most of the available therapies [1].

For symptomatic relief L-dopa in combination with carbidopa is the drug of choice apart from which dopamine agonists (DAs), catecolO-methyltransferase (COMT) inhibitors and monoamine oxidase inhibitors are the other drugs available. The marked difference in drug response and adverse effect profile among patients led to search of genetic markers and polymorphism associated with response to antiparkinsonian drugs which will enable us to predict an individual's response to drugs in terms of both efficacy and toxicity. Currently available drugs that target only the motor symptoms are ineffective lose over time and can cause catastrophic adverse effects. This variation in interindividual drug therapy has increased the need of personalized medicine and hence pharmacogenetic analysis of variations in drug response.

Pharmacogenomics is defined as the study of the genetic basis of drugs and drug therapy outcomes. Efficacy and toxicity are the two categories of treatment outcomes. Response to the same drug can vary among individuals, and significant differences are observed among individuals belonging to the same population as compared to within the same individual at different times [2]. About $20-95 \%$ of variations in drug pharmacokinetics and effects are estimated to be due to genetic factors. However, variation in genetic codes of drug metabolizing enzymes, transporters and receptors can also lead to interindividual variations [3]. An important aim of pharmacogenomics is to develop drugs with maximum efficacy with minimum toxicity by rationalized drug therapy [4]. "Personalized medicine" an upcoming concept, holds promise in that drugs and drug combinations are optimized for the genetic makeup of the individual [5]. Pharmacogenomics has wide applications in several diseases such as cardiovascular disease, psychosomatic and neurodegenerative disorders, chronic infectious diseases, metabolic disorders, and obstructive diseases of respiratory system.

Pharmacogenomics has the potential to change the way new drugs are developed, and the manner drugs are prescribed [6] via the use of genetic markers the drug response, its efficacy and potency can be predicted. Clinical trials will be designed to be enriched with individuals that are most in all likelihood to be benefitted by the drug, maximizing drug's efficacy, minimizing its unfavorable outcomes, and boosting the odds of successful discovery of drugs. Pharmacogenomics applied clinically will help physicians to accurately determine the right drugs, the right doses for right individuals [6]. It enables to avoid tedious trial and blunders approaches and keep away from detrimental impact consequently enhancing the patient compliance and improving the effectiveness of the therapy as well. Relatively, few efforts have been made to investigate the role of pharmacogenetics in anti-PD drug to evaluate individual response. Hence, many genetic variations and polymorphisms in a myriad of different proteins can affect person response to anti-PD drugs. Hence, we aim to present a critical review of the pharmacogenetic aspects of antiparkinsonian drug therapy and identify gene polymorphisms of interest for future research.

\section{PHARMACOGENOMICS AND PD}

Research performed in extraordinary settings propose that pharmacogenomics plays a widespread position in parkinsonism drug therapy. The gene/drug pairings with the most powerful capability 
for pharmacogenetic guidelines include: COMT allele/levodopa and entacapone, dopamine D2 receptor (DRD2)/ropinirole, pramipexole, and DRD3/ropinirole and pramipexole.

\section{TARGETS OF ANTIPARKINSONIAN TREATMENT}

\section{Dopamine receptors (DRDs)}

$\mathrm{PD}$ is the most recognized dopamine-related disorder, which originates from a loss of dopaminergic innervations in the brain. The physiological actions of dopamine are mediated by five different but intently related G protein-coupled receptors. The two major groups of which include: The D1 and D2 classes of DRDs [7]. Genetic cloning approaches later revealed that multiple receptor subtypes can be activated by dopamine. Based on structural, pharmacological, and biochemical properties, these receptors were classified as either D1-class DRDs (D1 and D5) or D2-class DRDs (D2, D3, and D4).

The individual members of the subfamilies of the D1- and D2-class receptors share an extensive level of homology of their transmembrane domains and feature distinct pharmacological properties. D1-class DRDs (D1 and D5) activates the $\mathrm{G} \alpha_{\mathrm{s} / \mathrm{lf}}$ family of $\mathrm{G}$ proteins to stimulate cyclic adenosine monophosphate formation using adenylcyclase. The D2-class DRDs couple to the $\mathrm{G} \alpha$, of $\mathrm{G}$ proteins and therefore result in inhibition of adenyl cyclase. Relative to the D1-class DRDs, D2, and D3 DRDs are expressed both postsynaptically on dopamine target cells and presynaptically on dopaminergic neurons [8].

The DRD1 and DRD2 are also extraordinary at the extent of genetic structure, typically inside the presence of introns in their coding sequences. The DRD1 and DRD5 genes do not comprise introns in their coding regions, however, the genes that encode the D2-class receptors have several introns, with six introns located in the gene that encodes the D2 dopamine receptor, 5 within the gene for the D3 dopamine receptor, and 3 in the gene for the DRD4 [9].

\section{GENE LOCATION AND ASSOCIATED POLYMORPHISM}

- The DRD1 gene is located on chromosome 5 and suggests numerous polymorphic loci that can modulate its feature like TaqIA polymorphism [10].

- The DRD2 gene encodes two isoforms with distinct functions, i.e., long (D2L) and short (D2S). It is located on chromosome 11. Numerous polymorphisms in DRD2 gene were discovered. The TaqIA polymorphism is related to decreased the range of striatal DRD2 receptor density in $A 1$ allele carriers and any other recognized polymorphism in DRD2 gene constitutes of repeat CA, DRD2 (CA)n, and is positioned in a non-coding location of the gene [11].

- The DRD3 gene is positioned on chromosome 3. Maximum investigated polymorphisms consist of Ser9Gly (or MscI or BalI), which may additionally affect DRD3 receptor membrane insertion, and its intracellular reaction [12]. DRD3 (rs6280) polymorphism has also been known to influence the age of onset in ethnic groups [13].

- The DRD4 gene is located on chromosome 11 and shows Variable number tandem repeats (VNTR) polymorphism inside $3^{\text {rd }}$ exon of 2-11 repeats of $48 \mathrm{bp}$, likely modulating G-protein binding [14].

- The DRD5 gene is located on chromosome 4 and its polymorphism might also, because of its similar shape and feature to DRD1, mediate antiparkinsonian drug actions.

\section{Dopamine active transporter (DAT)}

Uptake of dopamine into neurons is mediated by DAT. It is the main target for numerous pharmacologically active substances and environmental pollutants. The DA transporter 1 gene (DAT1, additionally referred to as SLC6A3) is a membrane-spanning protein that mediates the reuptake of dopamine from the synapse [15]. DAT1 is the number one regulator of dopamine neurotransmission and is expressed inside the central nervous system, primarily in areas that make up the dopaminergic circuits (e.g., striatum and nucleus accumbens). DAT1 carries variable variety tandem repeats (VNTRs), places within the genome in which a quick nucleotide series is repeated a number of instances. Special individuals may have an exclusive variety of repeats, which can cause the gene to behave otherwise (e.g., produce more or less protein).

DAT gene (SLC6A3) is positioned on chromosome 5. A 40 bp variable range tandem repeats (VNTR) polymorphism within the 3'UTR was identified, and might have an effect on gene expression, accordingly dopamine uptake from the synaptic cleft. 40-bp VNTR of the DAT is a predictor for the incidence of psychosis or dyskinesia in L-dopa-treated patients [16].

\section{COMT}

The transfer of a methyl group from S-adenosylmethionine to catecholamines is catalyzed by COMT. This 0-methylation results in one of the primary degradative pathways of the catecholamine transmitters. In addition to its position in the metabolism of endogenous materials, COMT is important in the metabolism of catechol drugs used within the remedy of hypertension, allergies, and PD. COMT is located in different forms in tissues, a soluble COMT (S-COMT) form, and a membrane-sure (MB-COMT) form. The differences among S-COMT and MB-COMT are inside the $\mathrm{N}$-termini. Several transcript editions are shaped via the usage of opportunity translation initiation sites and promoters.

\section{GENE LOCATION AND POLYMORPHISM}

- Gene coding for COMT is placed on chromosome 22. The G to A transition at codon 158 (Val158Met; [rs4680]) is the most generally studied COMT polymorphism. This results in the substitution of methionine for valine, and become to start with related to low enzyme activity (COMT-L) because of its thermo liability, opposite to H (high activity) allele [17].

- COMT haplotypes describe enzymatic activity significantly. The three maximum not unusual haplotypes are composed of four COMT SNPs combinations: One in the S-COMT promoter region and in the $\mathrm{S}$ - and MB-COMT - two synonymous change (rs4633:C>T, His62His) and (rs4818: C>G, Leu136Leu) in addition to one non-synonymous trade (rs4680:A>G, Val158Met) [17].

\section{HOMER1}

This gene encodes a member of the homer family of dendritic proteins. Contributors of this family alter institution 1 metabotropic glutamate receptor characteristics. It's miles a protein with the pivotal characteristic in glutamate transmission, which has been associated with the pathogenesis of levodopa-associated adverse drug effects.

\section{ORGANIC CATION TRANSPORTER (OCT)}

OCTs are involved inside the carriage of each endogenous compounds, together with dopamine, and drugs, like metformin and a few antiparkinsonian drugs. The three maximum important members of the OCT own family are OCT1, OCT2, and OCT3, encoded via the SLC22A1, SLC22A2, and SLC22A3 genes, respectively. Levodopa is a substrate for OCT, but the subtype is not always recognized. Pramipexole and amantadine are substrates for OCT1 and OCT2.

\section{PHARMACOGENETIC CRITERIA OF THE TREATMENT}

\section{Levodopa}

L-dopa is the best drug in the alleviation of motor impairments in PD, and its effectiveness and tolerability notably stepped forward after an introduction of its combination with a dopa-decarboxylase inhibitor, either benserazide or carbidopa. 50\% of PD patients dealt with l-dopa increase motor complications within 5 years after therapy initiation, and the threat yields $90 \%$ with the treatment longer than 10 years. However, a huge inter-person variability has been seen in PD patients with l-dopa therapy, both in terms of drug efficacy and toxicity, with the notable contribution of genetic factors, specifically in genes encoding drug receptors, metabolizing enzymes and intracellular signaling proteins.

The gene polymorphism affecting therapy is as shown in Table 1. 
Table 1: Gene polymorphisms affecting levodopa therapy



COMT: Catecol-0-methyltransferase

Table 3: Polymorphisms affecting DA therapy

\begin{tabular}{ll}
\hline Gene polymorphism & Effect observed \\
\hline Ser9Gly DRD3 polymorphisms: Ser/Ser>Gly/Gly & Significantly higher response rates to pramipexole \\
DRD2 rs1800497(TaqIA), DRD3 rs6280(Mscl), DRD3 & No significant association with non-ergoline DA discontinuation \\
rs4646996(Mspl) & $\begin{array}{l}\text { Significantly related with decreased discontinuation of non-ergoline treatment } \\
\text { Absence of DRD2 } 15 \text { CA repeat allele }\end{array}$ \\
DRD2 Taq1A polymorphism & No significant association with pramipexole efficacy \\
\hline
\end{tabular}

DAs: Dopamine agonists, DRD2: Dopamine D2 receptor

\section{COMT inhibitors}

Inhibition of COMT with new era COMT inhibitors, entacapone (peripherally acting), and tolcapone (peripheral and centrally acting) are powerful adjuvant therapy in PD. The efficacy is assessed using their dose-dependent inhibition of COMT interest in erythrocytes and a substantial lower within the plasma levels of 3-0-methyldopa. Availability of levodopa in the brain is increased by increasing the elimination half-life and hence the duration of action. Clinically, the improved levodopa availability is seen as prolonged duration of dyskinesias in PD patients with end-of-dose fluctuations. Thus, COMT inhibitors are a beneficial adjunct to levodopa therapy in PD patients with end-of-dose fluctuations [21]. The following gene polymorphism shown in Table 2 affects the efficacy and adverse effects of the COMT inhibitors $[17,18]$.

\section{DAs}

DAs exert their antiparkinsonian effects by acting directly on DRD and mimicking the endogenous neurotransmitter. There are two subclasses of DAs: Ergoline and non-ergoline agonists. Both of these subclasses target dopamine $\mathrm{D}_{2}$-type receptors. They were introduced as an adjunct to levodopa treatment in patients exhibiting fluctuating motor responses and dyskinesias associated with its chronic use. DAs have also been successfully used as monotherapy in de novo patients with the aim of delaying treatment with levodopa and therefore deferring the onset of complications [22].

The gene polymorphisms noted are as shown in Table 3 [23].

\section{CONCLUSION}

It can be concluded that with the rapid development of genotyping platforms, genome-wide association study can be performed to analyze polymorphism associated with inefficient treatment or adverse effects. Hence, individualized therapy of PD for better patient care can be achieved by targeted genetic testing.

\section{REFERENCES}

1. Factor SA. Current status of symptomatic medical therapy in Parkinson's disease. Neurotherapeutics 2008;5(2):164-80.

2. Vesell ES. Pharmacogenetic perspectives gained from twin and family studies. Pharmacol Ther 1989;41(3):535-52.

3. Kalow W, Tang BK, Endrenyi L. Hypothesis: Comparisons of inter- and intra-individual variations can substitute for twin studies in drug research. Pharmacogenetics 1998;8(4):283-9.

4. Sachidanandam R, Weissman D, Schmidt SC, Kakol JM, Stein LD, Marth G, et al. A map of human genome sequence variation containing 1.42 million single nucleotide polymorphisms. Nature 2001;409(6822):928-33.

5. Evans WE, Relling MV. Moving towards individualized medicine with pharmacogenomics. Nature 2004;429(6990):464-8.

6. Payami H, Factor SA. Promise of pharmacogenomics for drug discovery, treatment and prevention of Parkinson's disease. A perspective. Neurotherapeutics 2014;11(1):111-6.

7. Vallone D, Picetti R, Borrelli E. Structure and function of dopamine receptors. Neurosci Biobehav Rev 2000;24(1):125-32.

8. Rondou P, Haegeman G, Van Craenenbroeck K. The dopamine D4 receptor: Biochemical and signalling properties. Cell Mol Life Sci 2010;67(12):1971-86 
9. Beaulieu JM, Gainetdinov RR. The physiology, signaling, and pharmacology of dopamine receptors. Pharmacol Rev 2011;63(1):182-217.

10. Lee SP, So CH, Rashid AJ, Varghese G, Cheng R, Lança AJ, et al. Dopamine $\mathrm{D} 1$ and $\mathrm{D} 2$ receptor Co-activation generates a novel phospholipase C-mediated calcium signal. J Biol Chem 2004;279(34):35671-8.

11. Wang J, Liu ZL, Chen B. Association study of dopamine D2, D3 receptor gene polymorphisms with motor fluctuations in PD. Neurology 2001;56(12):1757-9.

12. Sokoloff P, Giros B, Martres MP, Bouthenet ML, Schwartz JC Molecular cloning and characterization of a novel dopamine receptor (D3) as a target for neuroleptics. Nature 1990;347(6289):146-51.

13. Hassan A, Heckman MG, Ahlskog JE, Wszolek ZK, Serie DJ, Uitti RJ, et al. Association of Parkinson disease age of onset with DRD2, DRD3 and GRIN2B polymorphisms. Parkinsonism Relat Disord 2016;22:102-5

14. Van Tol HH, Wu CM, Guan HC, Ohara K, Bunzow JR, Civelli O, et al. Multiple dopamine D4 receptor variants in the human population. Nature 1992;358(6382):149-52

15. Ciliax BJ, Heilman C, Demchyshyn LL, Pristupa ZB, Ince E, Hersch SM, et al. The dopamine transporter: Immunochemical characterization and localization in brain. J Neurosci 1995; 15:1714-23.

16. Kaiser R, Hofer A, Grapengiesser A, Gasser T, Kupsch A, Roots I, et al. $\mathrm{L}$-dopa-induced adverse effects in PD and dopamine transporter gene polymorphism. Neurology 2003;60(11):1750-5.

17. Lotta T, Vidgren J, Tilgmann C, Ulmanen I, Melén K, Julkunen I, et al. Kinetics of human soluble and membrane-bound catechol O-methyltransferase: A revised mechanism and description of the thermolabile variant of the enzyme. Biochemistry 1995;34(13):4202-10.

18. Contin M, Martinelli P, Mochi M, Riva R, Albani F, Baruzzi A. Genetic polymorphism of catechol-O-methyltransferase and levodopa pharmacokinetic-pharmacodynamic pattern in patients with Parkinson's disease. Mov Disord 2005;20(6):734-9.

19. Schumacher-Schuh AF, Altmann V, Rieck M, Tovo-Rodrigues L, Monte TL, Callegari-Jacques SM, et al. Association of common genetic variants of HOMER1 gene with levodopa adverse effects in Parkinson's disease patients. Pharmacogenomics J 2014;14(3):289-94.

20. Becker ML, Visser LE, van Schaik RH, Hofman A, Uitterlinden AG, Stricker BH. OCT1 polymorphism is associated with response and survival time in anti-Parkinsonian drug users. Neurogenetics 2011;12(1):79-82.

21. Ruottinen HM, Rinne UK. COMT inhibition in the treatment of Parkinson's disease. J Neurol 1998;11(3):25-34.

22. Brooks DJ. Dopamine agonists: Their role in the treatment of Parkinson's disease. J Neurol Neurosurg Psychiatry 2000;68(6):685-9.

23. Arbouw ME, Movig KL, Egberts TC, Poels PJ, van Vugt JP, Wessels JA, et al. Clinical and pharmacogenetic determinants for the discontinuation of non-ergoline dopamine agonists in Parkinson's disease. Eur J Clin Pharmacol 2009;65(12):1245-51. 